\title{
¿POR QUÉ TRABAJO SOCIAL? \\ VOCES DE ESTUDIANTES MEXICANOS EN NUEVO LEÓN \\ WHY STUDY SOCIAL WORK? \\ MEXICAN STUDENT VOICES IN NUEVO LEÓN
}

\author{
Martha Leticia Cabello Garza (1) \\ Luis M. Rodríguez Otero (2) \\ (1) Universidad de Nuevo León (2) Universidad Internacional de la Rioja
}

Resumen: Este trabajo presenta los resultados de una investigación que tuvo como objetivo identificar los motivos por los que un estudiante elige la profesión de trabajo social y conocer si la influencia de los diversos contextos del entorno pudiera ser determinante de esta elección. A través de un muestreo por conveniencia, se aplicó una encuesta sociodemográfica anónima a 159 estudiantes de trabajo social en México, de diferentes cursos, preguntándoles sobre los motivos por los que se eligió esta profesión. Se realizó un análisis mixto identificando categorías emergentes, analizando las frecuencias de las respuestas y la existencia de correlaciones respecto a las variables. La teoría de los imaginarios sociales ayudó a entender esos motivos. Influencias de la familia y el círculo social, contacto previo con trabajadores sociales, preocupación social por determinadas situaciones, así como algunas cuestiones personales fueron algunos motivos de la elección.

Palabras clave: Elección profesional, Trabajo social, Imaginarios sociales, Estudio mixto.

Abstract: This paper presents the findings of a study that aimed to identify the motives for which a student might choose the social work profession and find out if the influence of various contexts in his/her surroundings play a determining role in this choice. Using a convenience sample, an anonymous social-demographic survey was administered to 159 social work students in Mexico, in different years of the programme, asking them about their motives for choosing this profession. A combined analysis was performed to identify emerging categories, analyse response frequency and to detect any correlations between variables. The theory of social imaginaries helped the researchers understand these motives. The influence of the family and the social circle, previous contact with social workers, concern about certain social issues, and some personal questions were among the motives behind the choice.

Key words: Professional choice, Social work, Social imaginaries, Combined study.

| Recibido: 25/11/2016 | Revisado: 08/01/2017 | Aceptado: 17/01/2017 | Publicado: 31/01/2017 |

Correspondencia: Martha Leticia Cabello Garza. Docente-investigadora de la Facultad de Trabajo Social y Desarrollo Humano de la Universidad Autónoma de Nuevo León (México). E-mail: marthacabello@hotmail.com. Luis M. Rodríguez Otero. Profesor Asociado de la Universidad Internacional de la Rioja (UNIR) e investigador postdoctoral visitante del Centro de Estudios Sociales de la Universidad de Coimbra.). E-mail: luismaotero@yahoo.es.

Referencia normalizada: Cabello, M. L., y Rodríguez-Otero, L. M. (2017). ¿Por qué Trabajo Social? Voces de estudiantes mexicanos en Nuevo León. Trabajo Social Hoy, 80, 73-94. doi. 10.12960/TSH.2017.0005. 


\section{INTRODUCCIÓN}

Toda nuestra actividad se construye sobre un intercambio de informaciones que adquirimos en la vida diaria y que no necesitan ser organizadas de forma demasiado complicada. Sin embargo existen actividades en las que las informaciones adquieren una gran importancia sobre todo cuando se trata de elecciones que influyen o pueden ser determinantes en la vida. Una de ellas es la elección de la carrera.

Aunque pueden ser muy variados los porqués se toma la decisión de una carrera profesional, los motivos para elegir esta profesión siguen ligados a la intervención caritativa desde la época de la Corona española, donde la iglesia era la única forma de ayuda social y que continuó durante el período de independencia debido al caos político y social que prevalecía. Esto aunado a la incapacidad del gobierno de mejorar la situación y al surgimiento de instituciones públicas dependientes del gobierno hacia finales de los años treinta, lo cual tuvo una influencia favorable para el trabajo social, empezando a ser reconocida como profesión, jugando un rol muy importante en las primeras escuelas de trabajo social (Tello y Arteaga, 2000).

El trabajo social en México, se encuentra inmerso en una serie de situaciones críticas con grandes problemáticas sociales, políticas y económicas, contextualizadas en una enorme heterogeneidad geográfica, económica y cultural, así como por una gran diversidad étnica, lingüística y socioeconómica. Una gran parte de la población se encuentra en situación de vulnerabilidad y Nuevo León no está exento de ello. Es un Estado que se encuentra al norte de México, cuenta con una población de más de 5 millones de habitantes (INEGI [Instituto Nacional de Estadística y Geografía], 2015). La zona metropolitana de su capital Monterrey, se caracteriza por ser una zona industrializada y con mayor participación del sector terciario, donde los y las trabajadores/as sociales han ido incursionando más en el sector privado, de servicios, en el sector industrial y en organizaciones no gubernamentales, rompiendo con la tradición de incorporarse en mayor medida al ámbito público que, básicamente, y en la mayoría de los Estados de la República Mexicana, ha estado centrado en actividades dentro del área de asistencia social y del sector salud.

Para entender los motivos por los que estudiantes de la carrera de Trabajo Social y Desarrollo Humano optan por esta profesión, partimos del supuesto de que la elección profesional se construye a partir de imaginarios sociales. Según Cornelius Castoriadis (2007), vivimos en un mundo lleno de significaciones que van desde las que se institucionalizan hasta las que conllevan una fuerza imaginaria que se recrea y se construye de acuerdo a la realidad y a determinado contexto social. Lo cierto es que esta toma de decisiones se da de manera instantánea al terminar el nivel de preparatoria y es un proceso ligado a motivos conocidos o desconocidos por los estudiantes. 
Según Castoriadis (2007), el término de imaginarios sociales está asociado a acontecimientos socio-históricos, con formas de determinación social y procesos que los propios sujetos crean de su entorno, aseverando que las necesidades humanas son construcciones sociales que van cambiando en el tiempo y de acuerdo a la cultura en que se desarrollan. Así las instituciones como la iglesia, las escuelas, instituciones de salud, de asistencia social o de rehabilitación, incluso empresas privadas pueden ser comprendidas a partir de imaginarios sociales que se mueven entre la percepción de la realidad y la experiencia vivida en ellas.

De acuerdo con López Bonelli (1995, citada en Robles 2013), esta elección forma parte de un proceso que no se da por terminado con el ingreso a una universidad, sino que existen crisis vocacionales que suponen el reconocimiento de los gustos, intereses, clarificaciones de los motivos, de nuevos conocimientos, aptitudes, de imágenes de la realidad y la satisfacción en la elección. En el desarrollo de la elección profesional, de acuerdo a una investigación realizada por Raimundi, y otros (2006), se intersectan tres contextos simultáneos: el contexto imaginario, es decir, lo que debo hacer en términos de lo que los otros esperan que sea; el contexto simbólico, donde se construye la pregunta qué quiero ser y el contexto real que respondería a la pregunta dónde estoy y qué puedo ser. Inquietudes en el desempeño como docentes y los discursos de los estudiantes de trabajo social, vinculados con la identidad profesional, configuran enigmas que se intentan disipar en este artículo sobre los motivos que fundamentan la elección de una carrera.

Íntimamente ligada a la cuestión vocacional, se encuentra la identidad profesional y el posicionamiento que se pueda tener ante una disciplina. Hablar de identidad profesional de los/las trabajadores/as sociales remite necesariamente al concepto de vocación. Cabe mencionar que identidad vocacional e identidad profesional son diferentes términos, aunque muy parecidos, la primera expresa las variables de tipo afectivo motivacional, en tanto que la segunda, muestra el producto de la acción de determinado contexto sociocultural sobre la identidad vocacional. De acuerdo con Robles (2013, p. 54), frecuentemente utilizamos el término de vocación como "entrega", quizás desinteresada de algún oficio o profesión, pero para vincular el concepto de profesión al de vocación, podemos remitirnos a algunos postulados de Max Weber, quien sostiene que "la idea que representa el término da cuenta de considerar que el contenido más honroso del comportamiento moral es precisamente la conciencia del deber de la labor profesional en el mundo".

Oras posturas como las de Isuardi (citado por Robles, 2013), remiten a la vocación referida como un imaginario colectivo a través del cual un sistema recluta y organiza sus agentes de producción o como un "llamado", que utiliza el sistema para determinar el destino profesional. La relación existente entre el modo en que se concibe el ejercicio profesional nos llevó a cuestionarnos cuáles son los imaginarios sociales que los estudiantes tienen para la elección de esta profesión y si está siendo transformada o no a lo largo del proceso de su formación académica. 
Por otro lado Cegarra (2012, p. 12), afirma que más que significados, los imaginarios remiten a sentidos, es decir, "a múltiples significaciones que en conjunto conforman un marco de referencia o campo semántico que sirve de esquema de interpretación para comprender y aprehender la realidad socialmente dada". Desde este marco epistemológico resulta interesante conocer la gama de motivos por los que un estudiante de trabajo social elige esta profesión frente a una propuesta de un ciudadano asistido, beneficiario, cliente o usuario de un servicio, y ante un profesional del trabajo social "servidor", "salvador", "dador de ayuda", "virtuoso", agente de cambio", o "controlador de conflictos".

Los resultados de un estudio realizado por Robles (2013), en la Universidad de Buenos Aires (Argentina) acerca de la identidad del trabajador/a social que tienen los estudiantes acerca de esta profesión y los procesos de ciudadanía conformados por categorías éticas y políticas que giran alrededor de esta, podríamos afirmar que parece existir un impacto en las prácticas sociales previas a la formación académica, asumiendo que estas prácticas contribuyen a la conformación de ciertos imaginarios de las cosas y los hechos sociales, reforzando la idea de que la noción de trabajo social se construye socialmente.

Partiendo del hecho que la identidad profesional del trabajador social ha sido abordada desde diversos paradigmas, la elección de la carrera "se encuentra altamente polarizada entre los deseos de una inespecífica ayuda a los pobres (muchas veces originada en motivos de orden religioso) y el anhelo mesiánico de "cambiar el mundo"” (Robles, 2013, p. 18). En esta investigación, además, se encontró que algunas expectativas de los estudiantes de trabajo social están vinculadas por un lado a "cambiar el mundo", pero también señala que existen diferentes motivos por los que se elige ser trabajador social. Algunos de ellos son: los que están vinculados a la ayuda al necesitado. Otros de los motivos están vinculados a las cualidades de la carrera, tal es el caso del interés por el campo social, trabajo en conjunto, y otros más encaminados a cambiar y mejorar la situación social, resolver problemas, generar conciencia, hacer justicia o mejorar la calidad de vida de las personas. O bien, vinculados a experiencias familiares, a familias vinculadas por algún motivo al trabajo social, o a la influencia con personas que estudian o han estudiado trabajo social.

No existiendo investigaciones contextualizadas en México respecto a los motivos por lo que los estudiantes de trabajo social han decidido cursar estos estudios se plantea esta investigación tomando como referencia la Facultad de Trabajo Social y Desarrollo Humano de la Universidad Autónoma de Nuevo León, cuyo objetivo general es identificar los motivos por lo que sus estudiantes toman la decisión de cursar esta licenciatura. Asimismo se proponen como objetivos específicos por un lado conocer el calado que tiene la influencia del entorno en la muestra y también analizar si las variables relativas a la ideología política, prácticas religiosas y contacto previo de él/ella o su familia con algún trabajador/a social son determinantes en dichos motivos. 
Este trabajo permite buscar indagar respuestas a las interpretaciones que desde su percepción tienen los estudiantes y conocer si nuestro trabajo como docentes ha permitido ampliar el marco representacional basado en, no solo la idea de salvar o ayudar al mundo a resolver problemas sociales, sino ver qué tanto estamos avanzando en trabajar con los usuarios y clientes en pro de su autonomía. Conocer qué tanto los orígenes del trabajo social escapan a las condiciones del contexto, a las crisis socioeconómicas, o de inseguridad y violencia que viven nuestras comunidades, y al mismo tiempo generar hipótesis sobre los imaginarios sociales vinculados a "la ayuda", que está presente en las elecciones vocacionales de los futuros profesionales del trabajo social, o desde un paradigma crítico reflexionar respecto al interés académico que debería tener la temática vocacional en razón de intereses colectivos que se ponen en juego en las elecciones de una determinada profesión.

\section{METODOLOGÍA}

Esta investigación está basada en una metodología mixta (cualitativa y cuantitativa) y un diseño empírico-descriptivo, partiendo del paradigma interpretativo (Sandín, 2003; Montero \& León, 2007), a través de encuesta por muestreo por conveniencia, orientado a la identificación en una población concreta: estudiantes de Trabajo Social y Desarrollo Humano de la UANL.

Respecto a la parte cuantitativa en cada estratificación se han expresado los resultados como: totales, media y porcentaje, así como las correlaciones bivariadas de Pearson significativas a nivel 0,01 y 0,05 producidas entre las distintas variables analizadas (sexo, edad, municipio, semestre, conocido/os trabajador/es sociales y contacto previo con algún trabajador/a social como usuario/a). Para realizar el análisis cuantitativo previamente se realizó un análisis cualitativo de contenido a través de la construcción de categorías axiales de los distintos motivos expuestos por la muestra.

Cabe destacar que este estudio es de tipo exploratorio focalizado en un ámbito geográfico concreto y determinado. Es por ello que las conclusiones a las que se han llegado tienen un alcance limitado, aunque suficiente para la reflexión profesional al respecto.

La muestra que participó en esta investigación está formada por un total de 159 estudiantes de la licenciatura en Trabajo Social de la UANL.

\section{Instrumentos:}

El instrumento utilizado para esta investigación ha sido una encuesta sociodemográfica anónima, de elaboración propia formada por 9 cuestiones (6 de tipo cerrado y 3 abiertas) referentes a la identificación de las principales características de la muestra 
(sexo, edad, semestre que cursa, municipio, conocimiento de trabajadores/as sociales y vinculación como usuario/a de intervenciones desde el trabajo social). Asimismo se ha planteado una cuestión de tipo abierto referente a la identificación por parte de los participantes respecto a los motivos por los que decidieron estudiar trabajo social. No se ha establecido ninguna restricción al respecto y se han incluido la totalidad de los instrumentos.

\section{Procedimiento y codificación de resultados:}

El primer acercamiento consistió en la selección de siete grupos de la Licenciatura en Trabajo Social de la UANL. Posteriormente se acudió a las aulas para presentar la investigación y solicitar la participación del alumnado, informando previamente sobre el anonimato del instrumento (asegurando la confidencialidad) y el posterior análisis y publicación del estudio (respetando así las cuestiones éticas de la investigación). Los mismos investigadores que contactaron con la muestra aplicaron el instrumento de forma grupal durante el mes de enero de 2016 (Noreña, Alcázar-Moreno, Rojas \& Rebolledo-Malpica, 2012).

Respecto a la pregunta de tipo abierta planteada, se realizó la trascripción literal en documento Word de las respuestas. A continuación se procedió al análisis de contenido para categorizar las distintas manifestaciones expuestas por la muestra. Respecto al análisis de contenido realizado, se establecieron las siguientes fases: (i) codificación; (ii) definición del sistema de categorías y (iii) análisis e interpretación de datos. En la codificación en cada una de las categorías se han indicado: la edad $(\mathrm{E})$; sexo $(\mathrm{H}$-hombres y M-mujeres); el municipio (iniciales); si conoce a algún trabajador/a social (Si-No) y línea o líneas (I.) donde se expresa tal manifestación. Respecto a la fase de análisis e interpretación de datos se tomó como referencia el procedimiento de Colás (1998): (i) reducción de los datos; (ii) disposición y (iii) transformación de los datos, obtención y verificación de conclusiones. Para garantizar la calidad de la información obtenida se ha utilizado los criterios de confirmabilidad y neutralidad descritos por Noreña, AlcázarMoreno, Rojas \& Rebolledo-Malpica (2012): transcripciones textuales de la cuestión y contrastación de los resultados con la literatura existente.

Obtenidas las categorías se procedió a su codificación, así como respecto a las cuestiones sociodemográficas, en un programa estadístico (SPSS versión 20.0) y se realizó el tratamiento estadístico. Realizando un análisis respecto a qué categorías se evidenciaban en la muestra y respecto a qué variables se producen correlaciones significativas. Asimismo, tomando en consideración las manifestaciones de la muestra se expuso un ejemplo de cada categoría identificada, utilizando los criterios anteriormente expuestos de Colás (1998) y Noreña, Alcázar-Moreno, Rojas \& Rebolledo-Malpica (2012). 


\section{RESULTADOS}

A continuación se presentan los resultados divididos en varios apartados:

Características sociodemográficas de la muestra:

La muestra que participó en este estudio estaba formada por 159 alumnos/as de la Facultad de Trabajo Social y Desarrollo Humano de la Universidad Autónoma de Nuevo León (México), de los cuales 4 eran hombres (2.5 \%) y 155 mujeres (97.5\%) de edades comprendidas entre los 17 y los 43 años (media 19.6 años). Respecto al municipio de procedencia: 33 eran de Monterrey (20.8\%), 21 de San Nicolás (13.2 \%), 1 de San Pedro (0.6 \%), 26 de Apodaca (16.4 \%), 13 de Escobedo (8.2 \%), 21 de Guadalupe (13.2 \%), 6 de Juárez (3.8\%), 5 de Santa Catarina (3.1\%), 3 de municipios que no pertenecen al Área Metropolitana de Monterrey (1.9\%) y 30 no contestaron a la cuestión (18.9 \%).

Por otro lado respecto al semestre que estaban cursando: 60 estaban en $1^{\circ}(37.7 \%)$, 55 en $2^{\circ}$ (34.6\%), 24 en $6^{\circ}$ (15.1\%), 1 en $8^{\circ}$ (0.6 \%) y 19 en $9^{\circ}$ (11.9\%). Asimismo se observa que 128 (80.5 \%) señalaron que conocían a algún trabajador/a social y 47 (29.6 \%) que en alguna ocasión un trabajador/a social intervino con ellos/as.

\section{Análisis cualitativo y cuantitativo:}

A través del análisis de contenido de las respuestas de la muestra ante la cuestión referente a los motivos por los que habían decido estudiar Trabajo Social se han identificado un total de 6 categorías relativas a: (i) la intervención; (ii) cuestiones de ámbito social; (ii) el trabajo y/o el empleo; (iv) la influencia del entorno; (v) cuestiones académicas y (v) cuestiones personales (ver Tabla 1).

La primera categoría identificada, "intervención”, hace referencia a distintos aspectos relacionados con la práctica profesional del/de la trabajador/a social. Dentro de esta categoría las manifestaciones de la muestra se han agrupado en 14 subcategorías. La primera de ellas hace referencia a expresiones a través de las cuales indican que se decantaron por este tipo de estudio debido a los gustos que tienen por la intervención pero sin especificar un aspecto concreto.

Asimismo se aprecian cuestiones que hacen referencia a motivaciones referentes a la afinidad con actividades a través de las cuales se presta apoyo, ayuda, asesoría, motivación, orientación o capacitación a otras personas. También se identifican motivaciones relativas a la gestión de recursos, así como a actividades de prevención, solución de problemas e intervención con casos. Finalmente, dentro de esta categoría, se identifican cuestiones relativas a la intervención personal a través de la cual se desea aportar o contribuir a la profesión, el ejercicio del desarrollo humano o a intervenciones denominadas por los participantes como humanistas (ver Tabla 2). 
Tabla 1. Categorías y subcategorías identificadas

\begin{tabular}{|c|c|c|c|}
\hline 1. & Intervención & 4. & Influencia entorno \\
\hline 1.1. & General no especificado & 4.1. & Recomendación \\
\hline 1.2. & Apoyo & & 4.1.1. General no especificado \\
\hline 1.3. & Gestión & & 4.1.2. Amistades \\
\hline 1.4 . & Ayuda & & 4.1.3. Familia \\
\hline 1.5. & Prevención & & 4.1.4. Test vocacional \\
\hline 1.6. & Asesoría & 4.2. & Familia \\
\hline 1.7. & Motivación & & 4.2.1. Dar ejemplo a la familia \\
\hline 1.8. & Orientación & & 4.2.2. Obligación familiar \\
\hline 1.9. & Capacitación & & 4.2.3. Problemas económicos familiares \\
\hline 1.10 . & Solución de problemas & & 4.2.4. Antecedentes familiares trabajadores sociales \\
\hline 1.11 . & Aportar a la profesión & 4.3. & Círculo social \\
\hline 1.12. & Desarrollo humano & & 4.3.1. Amistades trabajadores sociales \\
\hline 1.13. & Humanismo & & 4.3.2. Contacto con trabajadores sociales \\
\hline 1.14. & Casos & & \\
\hline & & 5. & Cuestiones académicas \\
\hline 2. & Social & 5.1. & $2^{\mathrm{a}}$ opción estudios \\
\hline 2.1. & Preocupación social & 5.2. & Estudios previos relacionados \\
\hline 2.2. & Justicia social & 5.3. & Complemento formación previa \\
\hline 2.3. & Cambio/transformación social & 5.4 . & Aumento conocimiento \\
\hline 2.4. & Contribución & 5.5. & Importancia profesión \\
\hline & & 5.6. & Relación con Psicología \\
\hline 3. & Trabajo/empleo & & \\
\hline 3.1. & Trabajo con personas & 6. & Cuestiones personales \\
\hline 3.2. & Trabajo en comunidad & 6.1. & Vocación \\
\hline 3.3. & Trabajo en equipo & 6.2 & Superación \\
\hline 3.4. & Dirigir grupos & 6.3. & Suerte/azar \\
\hline 3.5. & Funciones & 6.4. & Habilidades sociales \\
\hline 3.6. & Distintos espacio de trabajo & & 6.4.1. Existencia no especificadas \\
\hline 3.7. & Mejora laboral & & 6.4.2. Mejora \\
\hline 3.8. & Empleabilidad & & 6.4.3. Paciencia \\
\hline 3.9. & Autoempleo & & 6.4.4. Escucha \\
\hline 3.10. & Sueldo & & 6.4.5. Confianza \\
\hline 3.11 . & Docencia & & 6.4.6. Hablar \\
\hline
\end{tabular}

Fuente: Elaboración propia.

Tabla 2: Categoría intervención: ejemplo, $n^{\circ}$. de participantes que la indican

\begin{tabular}{|l|l|l|c|}
\hline Subcategoría & Ejemplo: Cita & Identificador & $\begin{array}{c}\text { N. }{ }^{\circ} \text { participantes } \\
\text { identificado }\end{array}$ \\
\hline $\begin{array}{l}\text { General no } \\
\text { especificado }\end{array}$ & $\begin{array}{l}\text { "Me gusta escuchar, ayudar e } \\
\text { intervenir" }\end{array}$ & TS27, Mnc, S2, Si, I.12 & 2 \\
\hline Apoyo & $\begin{array}{l}\text { "Me gusta apoyar a las personas } \\
\text { que estén pasando por una } \\
\text { situación difícil" }\end{array}$ & TS1, M17, S2, Si, I.11 & 11 \\
\hline
\end{tabular}


¿Por qué trabajo social? Voces de estudiantes mexicanos en Nuevo León

Tabla 2: Categoría intervención: ejemplo, nº de participantes que la indican (Continuación)

\begin{tabular}{|c|c|c|c|}
\hline Subcategoría & Ejemplo: Cita & Identificador & $\begin{array}{l}\text { N. }{ }^{\circ} \text { participantes } \\
\text { identificado }\end{array}$ \\
\hline Gestión & $\begin{array}{l}\text { "Buscar el bienestar de la sociedad, } \\
\text { intervención en la sociedad, así } \\
\text { como también la orientación a las } \\
\text { personas, buscar soluciones y } \\
\text { planificar proyectos" }\end{array}$ & $\begin{array}{l}\text { TS153, M10, S6, Si, I. } \\
\text { 11-12 }\end{array}$ & 2 \\
\hline Ayuda & $\begin{array}{l}\text { "Me gustaba mucho cómo ayudaba } \\
\text { a los demás (una trabajadora social) } \\
\text { y yo quería hacer lo mismo" }\end{array}$ & TS2, M18, S2, Si, I.14-15 & 40 \\
\hline Prevención & $\begin{array}{l}\text { "Al principio porque pensaba que } \\
\text { era ayudar a la gente en algún tipo } \\
\text { de problema, pero me hicieron ver } \\
\text { que es más que eso, además de } \\
\text { ayudarlos con el problema, se debe } \\
\text { evitar problemáticas" }\end{array}$ & TS4, M20, S2, No, I.13-14 & 1 \\
\hline Asesoría & $\begin{array}{l}\text { "Decidí investigar sobre la carrera } \\
\text { ya que siempre quise trabajar } \\
\text { ayudando o asesorando a la gente } \\
\text { con problemas" }\end{array}$ & $\begin{array}{l}\text { TS38, Mnc, S2, No, I. } \\
13-14\end{array}$ & 1 \\
\hline Motivación & $\begin{array}{l}\text { "Me gusta trabajar con personas, } \\
\text { se me facilita escucharlas, me gusta } \\
\text { motivar a la gente" }\end{array}$ & TS72, Mnc, S9, Si, I.13-14 & 3 \\
\hline Orientación & $\begin{array}{l}\text { "El poder ayudar u orientar a los } \\
\text { demás es algo que me gusta" }\end{array}$ & TS5, M19, S2, Si, I. 15 & 28 \\
\hline Capacitación & $\begin{array}{l}\text { "Porque me interesan las } \\
\text { comunidades con problemas } \\
\text { sociales, el orientar y el capacitar } \\
\text { a las personas, así como ser } \\
\text { intermediaria en todo tipo de } \\
\text { situación a resolver" }\end{array}$ & TS75, M10, S1, Si, I.14-15 & 1 \\
\hline $\begin{array}{l}\text { Solución de } \\
\text { problemas }\end{array}$ & $\begin{array}{l}\text { "Con el fin de saber qué pasaba } \\
\text { más allá de las actitudes de las } \\
\text { personas, ¿por qué lo hacen? y de } \\
\text { qué forma se pueden solucionar } \\
\text { esos problemas" }\end{array}$ & TS60, M20, S9, Si, I.12-13 & 19 \\
\hline $\begin{array}{l}\text { Aportar a la } \\
\text { profesión }\end{array}$ & $\begin{array}{l}\text { "Porque me gusta aportar de mí } \\
\text { a la profesión, apoyar, buscar } \\
\text { soluciones a un problema que se } \\
\text { presente" }\end{array}$ & TS128, M17, S1, Si, I.11 & 1 \\
\hline $\begin{array}{l}\text { Desarrollo } \\
\text { Humano }\end{array}$ & "Por el desarrollo humano" & TS84, M24, S1, Si, I.12 & 2 \\
\hline Humanismo & $\begin{array}{l}\text { "Yo decidí elegir la carrera de } \\
\text { trabajo social ya que es muy } \\
\text { humanista" }\end{array}$ & TS109, M18, S1, Si, I.12 & 5 \\
\hline Casos & "Trabajar en trabajo de casos" & TS1, M17, S2, Si, I.13 & 1 \\
\hline
\end{tabular}

Fuente: Elaboración propia. 
Se observa que las subcategorías que tienen una mayor presencia en las manifestaciones de la muestra (ver Tabla 2) son las relativas a intervenciones a través de las cuales se presta ayuda a otras personas $(25,15 \%)$, seguido de intervenciones de orientación (17,61 \%), capacitación de usuarios/as (11,94\%), intervenciones de apoyo (6,91\%) e intervenciones definidas por los participantes como humanistas (3,14 \%). Asimismo se observa que en menor medida se hace referencia a cuestiones como las relativas a la motivación de los/as usuarios/as (1,88 \%), intervenciones no especificadas, de gestiones y/o definidas como desarrollo humano (1,25\% respectivamente), así como intervenciones de prevención, asesoramiento, capacitación y aportaciones a la profesión (0,6 2\% en cada una de ellas).

Si bien se ha revestido la disciplina del Trabajo Social de una serie de atributos, tratando de identificarla como ayuda a los pobres, orientador de conflictos o potenciadores de cambio, como afirman Bermúdez y colaboradores, (2006), estos estereotipos con los que se les ha etiquetado a lo largo del tiempo, han contribuido a desvirtuar el objeto que inspiró su institucionalización como profesión, que aunado al soporte que tiene su formación académica generando objetos específicos que no son exclusivos de su quehacer, perturba su identidad y la razón de ser.

Por otro lado se han identificado respuestas que mencionan cuestiones relativas a motivos vinculados a la preocupación social por determinadas situaciones o problemáticas existentes, a la justicia social derivada de dichas situaciones, al deseo de realizar contribuciones que reviertan en el entorno social y a la afinidad con el ejercicio de prácticas que tengan como fin producir un cambio social o la transformación social. Como todas estas cuestiones tienen como denominador común el impacto social se han aglutinado bajo la categoría de "cuestiones de ámbito social" (ver Tabla 3).

Asimismo existen participantes que señalan que se decantaron por esta formación debido a cuestiones "laborales". A través de esta categoría se han agrupado manifestaciones que hacen referencia al trabajo con personas, con comunidades o en equipo, así como al deseo de desempeñar funciones asociadas al trabajo social, la dirección de grupos o los espacios laborales donde se puede ejercer la profesión. También se expresan cuestiones que hacen referencia a motivaciones relativas a la promoción o mejora laboral, conseguir un empleo, el deseo de iniciar una actividad laboral por iniciativa propia (autoempleo), desempeñar la docencia o debido a cuestiones salariales.

Como se observa en la tabla anterior, la subcategoría referente a la transformación o cambio social tiene un gran calado en la muestra (17,61\%). Asimismo se observa que el resto de subcategorías relativas a las cuestiones sociales no son especialmente señaladas por los/as participantes: justicia social (4,40 \%), contribución social (1,25 \%) y preocupación social $(0,62 \%)$. 
¿Por qué trabajo social? Voces de estudiantes mexicanos en Nuevo León

Tabla 3. Cuestiones sociales: ejemplo, $n .^{\circ}$ de participantes que la indican

\begin{tabular}{|c|c|c|c|}
\hline Subcategoría & Ejemplo. Cita & Identificador & $\mathrm{N}^{\circ}$ \\
\hline Casos & "...Trabajar en trabajo de casos" & $\begin{array}{l}\text { TS1, M17, } \\
\text { S2, Si, I.13 }\end{array}$ & 1 \\
\hline $\begin{array}{l}\text { Preocupación } \\
\text { social }\end{array}$ & $\begin{array}{l}\text { "Porque me preocupan los problemas de la sociedad, me gusta } \\
\text { ayudar a las demás personas cuando puedo y me gustaría que me } \\
\text { pagaran por ello" }\end{array}$ & $\begin{array}{l}\text { TS122, H18, } \\
\text { S1, No, I. } \\
\text { 9-10 }\end{array}$ & 1 \\
\hline Justicia social & $\begin{array}{l}\text { "Siempre me han interesado los problemas sociales y quiero } \\
\text { aportar mi granito de arena para disminuir a estos problemas, para } \\
\text { el bien de la sociedad. Además me interesan los problemas en } \\
\text { adolescentes, la justicia social. Odio las injusticias" }\end{array}$ & $\begin{array}{l}\text { TS40, Mnc, } \\
\text { S2, No, l. } \\
\text { 13-15 }\end{array}$ & 7 \\
\hline $\begin{array}{l}\text { Cambio/ } \\
\text { transformación } \\
\text { social }\end{array}$ & $\begin{array}{l}\text { "Me gustaría poner mi granito de arena para general un cambio } \\
\text { positivo en la sociedad y se transforme" }\end{array}$ & $\begin{array}{l}\text { TS33, M18, } \\
\text { S2, Si, I. } \\
\text { 17-18 }\end{array}$ & 28 \\
\hline Contribución & $\begin{array}{l}\text { "...Por el campo laboral que abarca, permite contribuir a la } \\
\text { sociedad" }\end{array}$ & $\begin{array}{l}\text { TS142, M20, } \\
\text { S6, Si, I. } 11\end{array}$ & 2 \\
\hline
\end{tabular}

Fuente: Elaboración propia.

Tabla 4. Categoría cuestiones laborales: ejemplo, $n .^{\circ}$ de participantes que la indican

\begin{tabular}{|l|l|l|c|}
\hline Subcategoría & \multicolumn{1}{|c|}{ Ejemplo. Cita } & \multicolumn{1}{|c|}{ Identificador } \\
\hline $\begin{array}{l}\text { Trabajo con } \\
\text { personas }\end{array}$ & $\begin{array}{l}\text { "Porque siempre me ha gustado trabajar con la } \\
\text { gente en todo tipo de problemas" }\end{array}$ & TS92, M17, S1, Si, I. 12 & 35 \\
\hline $\begin{array}{l}\text { Trabajo en } \\
\text { comunidad }\end{array}$ & $\begin{array}{l}\text { "Porque es una carrera donde se involucra } \\
\text { mucho a la comunidad" }\end{array}$ & TS5, M19, S2, Si, I. 13 & 1 \\
\hline Trabajo en equipo & "Porque me gusta el trabajo en equipo" & TS 115, M17, S1, Si, I. 11 & 2 \\
\hline Dirigir grupos & "y dirigir grupos (me gusta)" & TS72, Mnc, S9, Si, I. 13-14 & 1 \\
\hline Funciones & $\begin{array}{l}\text { "Porque es una carrera que me llama en todos } \\
\text { los sentidos, me gusta las funciones que realiza }\end{array}$ & TS57, M22, S9, Si, I. 12-13 & 3 \\
\hline $\begin{array}{l}\text { Distintos espacios } \\
\text { de trabajo }\end{array}$ & "Por las áreas en las que intervienen" & TS137, M20, S6, Si, I. 11 & 3 \\
\hline Mejora laboral & "Para mejorar en mi trabajo" & TS59, M27, S9, Si, I. 12 & 2 \\
\hline Empleabilidad & $\begin{array}{l}\text { "..Y aparte un trabajador social tiene más } \\
\text { oportunidades de empleo" }\end{array}$ & TS95, M20, S1, No, I. 13-14 & 2 \\
\hline Autoempleo & "..Para realizar mi propia institución de apoyo" & TS141, M19, S6, Si, I. 11 & 1 \\
\hline Sueldo & "Porque ganas buen sueldo" & TS79, M17, S1, Si, I. 13 13 \\
\hline Docencia
\end{tabular}

Fuente: Elaboración propia. 
Se observa que la subcategoría que tiene una mayor presencia en las manifestaciones de la muestra, como se observa en la tabla anterior, es la relativa al trabajo con las personas (22,01\%). No obstante se observa que existe una pluralidad de subcategorías agrupadas bajo la categoría axial de trabajo/empleo: funciones del trabajo social, espacios de trabajo y docencia ( $1,88 \%$ cada una), trabajo en equipo, mejora laboral y empleabilidad (1,25\% respectivamente), así como trabajo en comunidad, dirección de grupos, autoempleo y sueldo (0,62 \% cada subcategoría).

La cuarta categoría identificada hace referencia a cómo el entorno social de los participantes ha influenciado en ellos. Esta categoría se ha denominado como "influencia del entorno". Se observa que la influencia proviene de tres vías: recomendaciones de distintas personas, la familia y el círculo social. Se observa que parte de la muestra indica que se ha decantado por esta licenciatura debido a que ciertas personas no especificadas se la recomendaron, otras indican que las recomendaciones procedían de amistades, la familia o del ámbito escolar debido a la realización de test vocacionales.

Respecto a las cuestiones familiares se observa que existen estudiantes que indican que eligieron los estudios de trabajo social debido a cuestiones como: (i) el deseo de dar ejemplo a sus familiares; (ii) la obligación de sus progenitores a estudiar la licenciatura; (iii) problemas económicos familiares que le marcaron y (iv) la existencia de familiares trabajadores sociales.

Asimismo se observa que existen estudiantes que indican que el círculo social influenció en su decisión debido a cuestiones como la existencia de amistades que son trabajadores sociales o al hecho de haber tenido contacto con estos profesionales, bien sea de forma directa como usuario/a o indirecta (ver Tabla 5).

Se observa que las subcategorías que tienen una mayor presencia en las manifestaciones de la muestra son las relativas al contacto con trabajadores/as sociales, lo cual es evidenciado en 23 participantes (14,46\%), bien sea el contacto con familiares que ejercen esta profesión (6,28 \%), amistades (0,62 \%) o conocidos (7,54 \%). Por otra parte se observa que la subcategoría de recomendación es señalada por el 2,51 \% de la muestra, la familia y el círculo social por el 8,17 \% respectivamente (ver Tabla 5).

También se observa que la muestra indica que su decisión se debió a "cuestiones académicas". Dentro de esta metacategoría se identifican cuestiones a través de las cuales la muestra manifiesta que deseaban estudiar otra carrera universitaria (la mayor parte psicología) pero que como $2^{a}$ opción de estudios tenían trabajo social. También se observa que estudiantes indican que su decisión se debió a que deseaban complementar una formación previa o debido a que habían cursado previamente estudios relacionados. Sin embargo existen estudiantes que indican que su decisión se basó en la importancia que tiene la profesión de trabajo social, el deseo de aumentar sus conocimientos o debido a la relación que consideran que tiene con la psicología. 
¿Por qué trabajo social? Voces de estudiantes mexicanos en Nuevo León

Tabla 5. Categoría influencia entorno: ejemplo, $n .^{\circ}$ de participantes que la indican

\begin{tabular}{|c|c|c|c|c|}
\hline \multicolumn{2}{|c|}{ Subcategoría } & Ejemplo. Cita & Identificador & $\mathrm{N}^{\circ}$ \\
\hline \multirow{4}{*}{ Recomendación } & $\begin{array}{l}\text { General no } \\
\text { especificado }\end{array}$ & $\begin{array}{l}\text { "Porque me recomendaron la carrera de } \\
\text { acuerdo a mis características personales" }\end{array}$ & $\begin{array}{l}\text { TS43, M17, } \\
\text { S2, Si, I. } 11\end{array}$ & 1 \\
\hline & Amistades & $\begin{array}{l}\text { "Conocí esta carrera por una amiga, y } \\
\text { me explicó de qué se trataba y me la } \\
\text { recomendó" }\end{array}$ & $\begin{array}{l}\text { TS37, M18, } \\
\text { S2, No, l. } \\
15-16\end{array}$ & 1 \\
\hline & Familia & $\begin{array}{l}\text { "Porque mi mamá me dijo que era casi lo } \\
\text { mismo que psicología y me gusta tener } \\
\text { contacto con la gente" }\end{array}$ & $\begin{array}{l}\text { TS148, M20, } \\
\text { S6, Si, I. } \\
\text { 10-11 }\end{array}$ & 1 \\
\hline & Test vocacional & $\begin{array}{l}\text { "Entrando en preparatoria en la materia } \\
\text { de orientación vocacional me solicitaron } \\
\text { una tarea que consistía en realizar un test } \\
\text { vocacional y el resultado me daba como } \\
\text { opción Trabajo Social" }\end{array}$ & $\begin{array}{l}\text { TS39, M43, } \\
\text { S2, No, I. } \\
12-13\end{array}$ & 1 \\
\hline \multirow{4}{*}{ Familia } & $\begin{array}{l}\text { Dar ejemplo a la } \\
\text { familia }\end{array}$ & "Y para dar ejemplo a mi familia" & $\begin{array}{l}\text { TS25, M41, } \\
\text { S2, Si, I. } 12\end{array}$ & 1 \\
\hline & $\begin{array}{l}\text { Obligación } \\
\text { familiar }\end{array}$ & $\begin{array}{l}\text { "Mi madre me dijo que estudiara aquí y casi } \\
\text { me obligó" }\end{array}$ & $\begin{array}{l}\text { TS123, M19, } \\
\text { S1, Si, I. } 11\end{array}$ & 1 \\
\hline & $\begin{array}{l}\text { Problemas } \\
\text { económicos } \\
\text { familiares }\end{array}$ & $\begin{array}{l}\text { "Para sacar a delante a mi hijo por la } \\
\text { necesidad que tenemos" }\end{array}$ & $\begin{array}{l}\text { TS110, M17, } \\
\text { S1, Si, I. } \\
\text { 13-14 }\end{array}$ & 1 \\
\hline & $\begin{array}{l}\text { Antecedentes } \\
\text { familiares } \\
\text { trabajadores } \\
\text { sociales }\end{array}$ & $\begin{array}{l}\text { "Porque tengo una tía que es trabajadora } \\
\text { social y me contaba la manera en que } \\
\text { trabajaba y las cosas que hacía, me llamó la } \\
\text { atención y empecé a buscar más sobre la } \\
\text { carrera y elegí estudiar Trabajo Social" }\end{array}$ & $\begin{array}{l}\text { TS7, Mnc, } \\
\text { S2, Si, I. } \\
11-13\end{array}$ & 10 \\
\hline \multirow{2}{*}{ Círculo social } & $\begin{array}{l}\text { Amistades } \\
\text { trabajadores } \\
\text { sociales }\end{array}$ & $\begin{array}{l}\text { "Porque conocí a la hermana de una amiga } \\
\text { y me dijo de qué trataba la carrera y como } \\
\text { a mi me gustaba ayudar a las personas (...) } \\
\text { me decidí por estudiar trabajo social porque } \\
\text { sé que en algún futuro cumpliré ese sueño" }\end{array}$ & $\begin{array}{l}\text { TS56, M17, } \\
\text { S2, No, l. } \\
13-15\end{array}$ & 1 \\
\hline & $\begin{array}{l}\text { Contacto con } \\
\text { trabajadores } \\
\text { sociales }\end{array}$ & $\begin{array}{l}\text { "Porque me ha tocado convivir con una } \\
\text { trabajadora social (...) y porque nos ayuda } \\
\text { con la situación que tenemos con mi } \\
\text { abuelita" }\end{array}$ & $\begin{array}{l}\text { TS112, M } \\
18, \text { S1, Si, I. } \\
13-14\end{array}$ & 12 \\
\hline
\end{tabular}

Fuente: Elaboración propia. 
Tabla 6. Categoría cuestiones académicas: ejemplo, n. ${ }^{\circ}$ de participantes que la indican

\begin{tabular}{|c|c|c|c|}
\hline Subcategoría & Ejemplo. Cita & Identificador & $\mathrm{N}^{\circ}$ \\
\hline $2^{\mathrm{a}}$ opción estudios & $\begin{array}{l}\text { "Porque quería estudiar una licenciatura, pensé en } \\
\text { estudiar psicología, pero me incliné por trabajo social } \\
\text { porque no fui aceptada y por la labor que no se enfoca en } \\
\text { lo económico sino en la situación social" }\end{array}$ & $\begin{array}{l}\text { TS63, M22, S9, } \\
\text { Si, I. 12-13 }\end{array}$ & 7 \\
\hline $\begin{array}{l}\text { Estudios previos } \\
\text { relacionados }\end{array}$ & $\begin{array}{l}\text { "Durante los tres años que cursé la preparatoria (BT- } \\
\text { Trabajo Social) me di cuenta que es una profesión muy } \\
\text { linda, dedicada la sociedad, que hace todo lo posible por } \\
\text { brindar ayuda, apoyo a las personas más necesitadas, } \\
\text { y también que aparte de ayudar como la mayoría de las } \\
\text { personas lo ven, brindamos u ofrecemos las herramientas } \\
\text { necesarias para poder solucionar los conflictos que se } \\
\text { presentan cotidianamente" }\end{array}$ & $\begin{array}{l}\text { TS32, M19, S2, } \\
\text { Si, I. 12-16 }\end{array}$ & 8 \\
\hline $\begin{array}{l}\text { Complemento } \\
\text { formación previa }\end{array}$ & $\begin{array}{l}\text { "Decidí estudiar trabajo social porque es un complemento } \\
\text { a mi carrera de ingeniería" }\end{array}$ & $\begin{array}{l}\text { TS95, M20, S1, } \\
\text { No, I. } 12\end{array}$ & 1 \\
\hline $\begin{array}{l}\text { Aumento } \\
\text { conocimiento }\end{array}$ & $\begin{array}{l}\text { "Saber cómo intervenir con las personas va influenciadas, } \\
\text { saber más" }\end{array}$ & $\begin{array}{l}\text { TS126, M18, S1, } \\
\text { Si, I. } 12\end{array}$ & 5 \\
\hline $\begin{array}{l}\text { Importancia } \\
\text { profesión }\end{array}$ & $\begin{array}{l}\text { "Por la importancia que tiene el trabajo de los } \\
\text { trabajadores sociales" }\end{array}$ & $\begin{array}{l}\text { TS138, M18, S6, } \\
\text { Si, I. 11-12 }\end{array}$ & 1 \\
\hline $\begin{array}{l}\text { Relación con } \\
\text { Psicología }\end{array}$ & $\begin{array}{l}\text { "Porque la involucré con la psicología y me di cuenta de } \\
\text { que tiene mucho de ello" }\end{array}$ & $\begin{array}{l}\text { TS68, M25, S9, } \\
\text { Si, I. } 13\end{array}$ & 1 \\
\hline
\end{tabular}

Fuente: Elaboración propia.

Se observa que las subcategorías que tienen una mayor presencia en las manifestaciones de la muestra son las relativas a la realización de estudios previos relacionados con el trabajo social (5,03 \%), la decisión debido a que era la segunda opción ya que existía otro estudio al que no pudo acceder (4,40 \%) y al deseo de aumentar el conocimiento $(3,14 \%)$. Asimismo se observa que el resto de subcategorías las manifiestan el 0,62 \% de la muestra en cada una de ellas.

Finalmente se observa que existen manifestaciones que hacen referencia a "cuestiones personales", no clasificadas en las categorías anteriores. Dentro de este grupo axial se identifican cuestiones que hacen referencia a la idea de que tiene una vocación de trabajador/a social, motivaciones que hacen referencia a un proceso de superación personal, la suerte o el azar, así como a aspectos relativos a la existencia, adquisición, mejora o falta de habilidades sociales, tales como la escucha, la paciencia, la confianza o habilidades comunicativas. 
¿Por qué trabajo social? Voces de estudiantes mexicanos en Nuevo León

Tabla 7. Categoría cuestiones personales: ejemplo, $n .^{\circ}$ de participantes que la indican

\begin{tabular}{|c|c|c|c|c|}
\hline \multicolumn{2}{|c|}{ Subcategoría } & Ejemplo. Cita & Identificador & $\mathrm{N}^{\circ}$ \\
\hline \multicolumn{2}{|l|}{ Superación } & "Como por superación personal" & TS25, M41, S2, Si, I. 11 & 1 \\
\hline \multicolumn{2}{|l|}{ Suerte/azar } & $\begin{array}{l}\text { "Al momento de elegir no sabía muy } \\
\text { bien lo que era, fue una decisión } \\
\text { tomada muy a la ligera, corrí con la } \\
\text { suerte de que me encantó" }\end{array}$ & TS71, M21, S9, Si, I. 15-15 & 1 \\
\hline \multirow{6}{*}{$\begin{array}{l}\text { Habilidades } \\
\text { sociales }\end{array}$} & $\begin{array}{l}\text { Existencia no } \\
\text { especificadas }\end{array}$ & $\begin{array}{l}\text { "Porque creo tener las habilidades } \\
\text { sociales para su desempeño" }\end{array}$ & TS94, M18, S1, Si, I. 12 & 2 \\
\hline & Mejora & $\begin{array}{l}\text { "No soy muy buena con los grupos } \\
\text { numerosos y considero que en esta } \\
\text { carrera voy a aprender a trabajar y } \\
\text { adaptarme a lo que se me presente" }\end{array}$ & $\begin{array}{l}\text { TS151, M20, S6, Si, I. } \\
\text { 11-12 }\end{array}$ & 3 \\
\hline & Paciencia & $\begin{array}{l}\text { "Me gusta tratar a las personas, me } \\
\text { gusta ayudar y tengo paciencia" }\end{array}$ & TS51, M17, S2, Si, I. 11-12 & 1 \\
\hline & Escucha & $\begin{array}{l}\text { "y escuchar todo lo que me tengan } \\
\text { que decir (gustar), desde alegrías } \\
\text { hasta problemas o tristezas y daba } \\
\text { mi mejor consejo" }\end{array}$ & $\begin{array}{l}\text { TS101, M19, S1, Si, I. } \\
\text { 14-15 }\end{array}$ & 6 \\
\hline & Confianza & $\begin{array}{l}\text { "Para poder tener confianza con las } \\
\text { personas" }\end{array}$ & TS70, M23, S9, Si, I. 12 & 1 \\
\hline & Hablar & $\begin{array}{l}\text { "Porque me gusta dialogar con la } \\
\text { gente" }\end{array}$ & TS119, M17, S1, No, I. 11 & 1 \\
\hline
\end{tabular}

Fuente: Elaboración propia.

Como se observa en la tabla anterior las subcategorías que tienen una mayor presencia en las manifestaciones de la muestra son las relativas a la existencia de habilidades sociales denominadas por los/as participantes como escucha (3,77\%), así como el deseo de mejorar o adquirir habilidades sociales (1,88 \%) y habilidades no especificadas (1,25 \%). El resto de subcategorías son mencionadas por el 0,62 \% de la muestra respectivamente.

\section{Análisis cuantitativo:}

Tras realizar un análisis estadístico con objeto de identificar si se producen correlaciones bivariadas a nivel 0.01 y 0.05 entre las variables relativas al sexo, la edad, el semestre que cursa, el municipio, el conocimiento de trabajadores/as sociales y la 
vinculación como usuario/a de intervenciones desde el trabajo social y los motivos (categorías) por los que la muestra decidió estudiar trabajo social se observa que, como se expone en la siguiente tabla, se producen un total de 5 correlaciones, de las cuales 4 son a nivel 0.01 y 1 a nivel 0.05 y 2 directamente y 3 inversamente proporcionales.

Tabla 7. Análisis correlacional variables del estudio, medias y desviaciones típicas

\begin{tabular}{|c|c|c|c|c|c|c|c|c|c|}
\hline \multirow{2}{*}{\multicolumn{2}{|c|}{ Categoría }} & \multirow{2}{*}{ Media } & \multirow{2}{*}{ DT } & \multicolumn{6}{|c|}{ Correlaciones $^{1}$} \\
\hline & & & & Sexo & Edad & Semestre & Municipio & $\begin{array}{c}\text { Conoce } \\
\text { TS }\end{array}$ & $\begin{array}{c}\text { Intervino } \\
\text { TS }\end{array}$ \\
\hline \multicolumn{2}{|l|}{ Intervención } & 1.94 & 0.06 & 0.003 & 0.143 & $.278\left(^{\star \star}\right)$ & -0.072 & -0.131 & -0.137 \\
\hline \multicolumn{2}{|l|}{ Social } & 1.94 & 0.116 & 0.006 & 0.111 & 0.013 & $.168\left(^{\star}\right)$ & -0.061 & 0.074 \\
\hline \multicolumn{2}{|c|}{ Trabajo/empleo } & 0 & 0 & .(a) & .(a) & .(a) & .(a) & .(a) & .(a) \\
\hline \multirow{3}{*}{$\begin{array}{l}\text { Influencia } \\
\text { entorno }\end{array}$} & Recomendación & 1.9921 & 0.04 & -.029 & $-.254\left({ }^{\star \star}\right)$ & 0.033 & -0.045 & -0.093 & -0.117 \\
\hline & Familia & 1.9796 & 0.06 & 0.099 & -0.097 & 0.152 & -0.004 & 0.147 & -0.097 \\
\hline & Círculo Social & 1.9591 & 0.14 & -0.044 & 0.131 & 0.033 & $.176\left(^{\star}\right)$ & -0.025 & 0.044 \\
\hline \multicolumn{2}{|l|}{$\begin{array}{l}\text { Cuestiones } \\
\text { académicas }\end{array}$} & 1.97 & 0.058 & -0.066 & -0.036 & -0.095 & -0.102 & -0.023 & 0.110 \\
\hline \multirow{4}{*}{$\begin{array}{l}\text { Cuestiones } \\
\text { personales }\end{array}$} & Vocación & 1.97 & 0.157 & $\left..2311^{(\star *}\right)$ & 0.019 & -0.038 & 0.064 & 0.079 & 0.149 \\
\hline & Superación & 1.99 & 0.079 & -.013 & $-.567\left(^{\star \star}\right)$ & 0.032 & 0.038 & 0.039 & -0.052 \\
\hline & Suerte/azar & 1.99 & 0.079 & -.013 & -0.025 & $-.170\left(^{\star}\right)$ & -0.058 & 0.039 & 0.116 \\
\hline & $\begin{array}{l}\text { Habilidades } \\
\text { sociales }\end{array}$ & 1.98 & 0.045 & -.048 & 0.068 & 0.085 & -0.108 & 0.089 & -0.049 \\
\hline
\end{tabular}

$1\left(^{\star}\right)$ Correlación significativa a nivel 0.05; (*) Correlación significativa a nivel 0.01; (a) Una de las variables es constante.

Fuente: Elaboración propia.

Asimismo se observa que respecto a las variables relativas al conocimiento o contacto con trabajadores/as sociales no se produce ninguna correlación. Respecto a la categoría relativa a la "intervención" se produce una correlación directa con el semestre. Al igual que entre la categoría "social", y el municipio de la muestra. También se identifican 2 correlaciones inversamente proporcionales entre la edad y la influencia del entorno y las cuestiones personales (superación). Finalmente se identifica otra correlación directamente proporcional entre el sexo y cuestiones personales (vocación).

Cabe señalar que del total de correlaciones posibles en este análisis (66), solamente se produce en 5 de ellas (7.57 \%). No obstante se cerciora que el hecho de conocer a trabajadores/as sociales y la vinculación como usuario/a de intervenciones desde el trabajo social no resultan estadísticamente determinantes. 


\section{DISCUSIÓN}

Algunos estudiosos del tema como Montero (2000) desde un paradigma subjetivo mencionan algunas voces, significados y vivencias de la elección de una carrera profesional, reconociendo que las prácticas desarrolladas en torno a la elección de la carrera deben partir de una comprensión holística considerando tanto elementos personales como psicológicos. Esto cobra sentido en mayor proporción cuando se estudian carreras donde la personalidad y las habilidades de un profesional deben estar basadas en un perfil centrado en el interés hacia el ser humano, con capacidades de comunicación, empatía, liderazgo, como lo es la licenciatura en Trabajo Social y Desarrollo Humano.

Por otra parte Montero (2000), hace referencia a la articulación de la elección de la carrera por parte de los estudiantes con concepciones e imágenes sociales de éxito y con promesas académicas y profesionales que se han socializado en sus ambientes cercanos, pero que muchas veces está lejos de una realidad laboral y de oportunidades de empleo que mucho dependen de otras circunstancias. En nuestra localidad, aún se vive el llamado "compadrazgo", y el "nepotismo", donde el tener contactos personales o familiares con algún coordinador de área o en algún puesto político o de autoridad puede pesar más aún que las propias calificaciones, habilidades, preparación o méritos académicos. Imágenes sociales de éxito que algunas veces se derrumban en los egresados de la carrera.

Otro trabajo encontrado sobre la identidad profesional del trabajador social contemporáneo fue el de Morales y Torres (2010), quienes encontraron algunos significados sociales que los propios trabajadores sociales y la misma sociedad le otorgan a esta disciplina. Esto resulta interesante de revisar porque una de las categorías encontradas en este estudio y que constituye uno de los principales motivos por los que los estudiantes eligen la carrera es por las interacciones o experiencias previas de ellos mismos o de algún familiar con algún profesional del trabajo social.

En los hallazgos del estudio de Morales y Torres realizado en la Universidad Colegio Mayor de Cundinamarca (2010, p. 9), se muestra "un marcado imaginario social que etiqueta a la profesión como benefactora y paternalista", como se observa en la Tabla 2, las intervenciones a través de las cuales se presta ayuda a otras personas, es compartida por la mayoría de los participantes, y aunque se observan otras motivaciones para elegir la carrera con intervenciones de orientación, gestión, capacitación y otras, es importante sensibilizar a la población de que esta profesión trasciende a la ayuda paliativa y material, ya que estas acciones si bien son empleadas como respuestas inmediatas a una determinada situación, en nuestra localidad cada vez más, el profesional del trabajo social gana espacios en áreas empresariales encargadas de recursos humanos y en capacitación de personal, espacios en 
contextos políticos, o en el área de proyectos de investigación o de intervención en organizaciones no gubernamentales, pero no solo como encuestadores o visitadores sociales, sino como responsables de los mismos proyectos.

Otro estudio encontrado sobre las motivaciones de estudiantes universitarios de trabajo social de género masculino en la Universidad de Zulia fue el de Salazar y Rodríguez (2011), cuyo objetivo fue interpretar los motivos de ellos ligados a sentimientos y pensamientos del quehacer profesional. Resulta interesante conocer cómo a partir del mundo subjetivo de los estudiantes convergen metas y propósitos donde se involucra el crecimiento personal, académico y profesional. Las metas son relevantes para la mayor posibilidad de adquirir habilidades y aplicar conocimientos, pero las motivaciones sociales en este estudio surgen durante el proceso de formación académica a partir de su práctica profesional. Los resultados de esta investigación muestran similitudes en el trabajo de Salazar y Rodríguez, ya que a medida que transcurre la carrera los estudiantes fortalecen sus motivaciones que emergen del componente formativo de la carrera universitaria.

\section{CONCLUSIÓN}

Para dar respuesta a la pregunta central de esta investigación sobre los motivos que llevan a los estudiantes a elegir esta carrera profesional, se retomó la teoría de los imaginarios sociales, así como una revisión teórica del tema que fue contrastada o verificada a través de algunos estudios. Nos resultó interesante ver cómo el imaginario del trabajo social está presente en los discursos de las y los estudiantes de Trabajo Social, lo cual nos lleva a repensar qué tanto camino llevamos recorrido en dar a conocer nuestras fortalezas, y a su vez reconocer las debilidades profesionales, una de ellas es lo poco que los estudiantes consideran en la carrera la generación de conocimientos que sustenten teórica, epistemológica y metodológicamente nuestro actuar.

Si bien los motivos que orientan a los estudiantes de trabajo social a elegir la Licenciatura en Trabajo Social y Desarrollo humano, están dirigidos a la práctica profesional, principalmente centrados en la ayuda a personas vulnerables, a preocupaciones centradas en el cambio o transformación social, a funciones relacionadas con el ámbito laboral, habilidades sociales o motivaciones personales y situaciones del contexto social donde se insertan los profesionales del trabajo social, los resultados encontrados en la presente investigación nos señalan la necesidad que tienen los jóvenes de contar con espacios educativos que les permitan conocer no solo el perfil profesional de cada carrera sino los principios y valores que los rigen, pero además que les permita conocer de cada uno sus fortalezas y debilidades que les lleve a la toma de decisiones informada. 
La toma de decisiones informada para elegir una profesión y trazar un propósito de carrera es crucial en la vida de los estudiantes, que en sus primeros años se enfrentan a dudas sobre la profesión. Por otro lado es o debería ser una labor imprescindible de las materias de orientación en los niveles de educación media y media superior.

Experiencias personales con profesionales del trabajo social, influencia de otros para elegir la profesión como la familia o los amigos, o el hecho de que algún familiar estudie la carrera han sido elementos constantes en algunos estudios encontrados del tema (Montero, 2000; Raimundi, et al., 2006; Robles, 2013). Sin embargo debemos resaltar como preocupante que la elección de la carrera en algunos casos sea impuesta por alguno de los padres o la influencia de amigos, ya que para esta profesión se hace necesario contar con estudiantes y más aún de profesionistas dispuestos a enfrentar la complejidad de las nuevas y emergentes problemáticas sociales, afrontar la dinámica de la sociedad actual cada vez más cambiante e incierta.

Pero además esa desinformación puede traer algún grado de frustración y "desencanto social", de quienes no logran sus expectativas de ingresos o de ser contratados en el área que más les interese (Montero, 2000). Es pues imprescindible que se destine presupuesto para realizar programas en el campo de la orientación educativa, y no se esté apostando a obtener de los estudiantes el máximo aprovechamiento de sus capacidades y habilidades para el beneficio de su comunidad, ya que algunos estudiantes se ven obligados a obtener titulaciones sin vocación.

A su vez nos resulta preocupante el imaginario social de ayuda al necesitado, netamente bajo una concepción asistencialista donde las calificaciones y las oportunidades salariales y laborales futuras pasan a segundo plano o no aparecen. También es oportuno mencionar que en los imaginarios sociales que el estudiante tiene de la profesión, se denota un limitado conocimiento de las áreas y espacios de actuación, poco reconocimiento de los sujetos-objetos de intervención y de sus roles y funciones. Esto genera grandes interrogantes en términos de la visibilidad de nuestra profesión ante la sociedad y un débil posicionamiento que nos hace preguntarnos ¿por qué la profesión no se expresa?, ¿por qué en nuestra localidad no se lucha por obtener mejores salarios?

Nuestra propuesta versa en función de hacer visible la profesión del trabajo social exaltando la seguridad de ejercer la profesión dejando a un lado el hacer por hacer, que se traduce en una concepción del trabajo social que ha sido nociva en nuestro país y específicamente en nuestra entidad. Promulgamos promover las fortalezas de los profesionales, como la de desarrollar sus actividades siempre de manera ética y preservando los valores de justicia social, los derechos humanos, la responsabilidad colectiva y el respeto a la diversidad, tal como lo estipula la definición de trabajo social adoptada en la Conferencia Mundial sobre el Trabajo Social por la Federación Internacional de 
Trabajo Social (FITS, 2014) y así contribuir a replantear la profesión de manera urgente ante los nuevos retos que la sociedad nos demanda y a su vez cambiar la percepción que la sociedad tiene de esta disciplina.

Nuestra pretensión no es corroborar o refutar alguna teoría relacionada con la vocación de ser trabajador/a social, pero sí comprender los imaginarios sociales que existen en los estudiantes y que se generan en las interacciones con otros profesionales, con los usuarios y los significados relacionados con experiencias de intervención social propias que traen recuerdos y anticipaciones con ellos mismos o con su familia. Desde la teoría de los imaginarios sociales, la imagen que se tiene de los y las trabajadores/as sociales es un factor determinante en el pensamiento de los estudiantes y la actitud que ellos asumen para elegir esta profesión está determinada por esa construcción social. Ante este panorama mucho tenemos por hacer, como promover la visibilidad de un trabajo social renovado, científico, posicionado como una profesión de alto valor social ante los retos que nos impone la sociedad de hoy.

\section{REFERENCIAS BIBLIOGRÁFICAS}

Bermúdez, A.; Sánchez, C. M.; Bernal, M. E. \& Castaño, E. (2006). Reconocimiento del ser profesional del Trabajo Social. Revista Latinoamericana de Estudios Educativos, 2(2), 131-147.

Castoriadis, C. (2007). La institución imaginaria de la sociedad. Colección Ensayo. Buenos Aires, Argentina: Tusquets Editores.

Cegarra, J. (2012). Fundamentos Teórico Epistemológicos de los Imaginarios Sociales. Cinta moebio 43: 1-13 disponible en: www.moebio.uchile.cl/43/cegarra.html.

Colás, P. (1998). El análisis cualitativo de datos. En L. Buendía, P. Colás, \& F. Hernández, Métodos de investigación en Psicopedagogía (págs. 288-313). Madrid: McGraw-Hill Interamericana de España.

Federación Internacional de Trabajo Social (FITS, 2014). Proposed new global definition of Social Work. En: http://ifsw.org/get-involved/global-definition-of-social-work/

Instituto Nacional de Estadística y Geografía [INEGI] (2015). Población total en Nuevo León, cifras del 2015. Consultado el 16 de septiembre de 2016 en: http://www. inegi.org.mx/.

Montero, M. T. (2000). Elección de carrera profesional. Visiones promesas y Desafíos. México: Universidad Nacional Autónoma de México.

Montero, I., \& León, O. G. (2007). A guide for naming reseacher studies in Psychology. Instrumental Journal of Clinical and Heath Psychology, 7 (1), 847-862.

Morales, M. F., y Torres, V. L. (2010). Aproximaciones acerca de la identidad profesional del Trabajador Social contemporáneo. Margen 59,1-38.

Noreña, A. L., Alcázar-Moreno, N., Rojas, J., \& Rebolledo-Malpica, D. (2012). Aplicabilidad de los criterios de rigor y éticos en la investigación cualitativa. Aquichan, 12(3), 263-274. 
Raimundi, L., Seneglia, F. Kilduff, F., \& Maté, V. (2006). El Trabajo Social como elección vocacional. Escenarios 10. Buenos Aires, Argentina: Espacio Editorial.

Robles, C. (2013). Trabajo Social como elección profesional. Buenos Aires, Argentina. Espacio Editorial.

Salazar, E. M., \& Rodríguez, X. (2011). Motivaciones sociales en estudiantes universitarios de trabajo social, desde las vivencias de los jóvenes de género masculino. Omnia 17 (2), 71-90.

Sandín, M. (2003). Investigación cualitativa en educación: Fundamentos y tradiciones. Madrid: McGraw-Hill Interamericana de España.

Tello, N. y Arteaga, C. (2000). Historia del Trabajo Social en México. Notas para una discusión. En N. E. Tello (Coord.). Trabajo Social en Algunos países. Aportes para su comprensión. Cd de México: Universidad Autónoma de México. Escuela Nacional de Trabajo Social. 